\title{
THE EFFECT OF PLASMA DENSITY ON THE BEHAVIOR OF THE PLASMA BOUNDARY IN HELIUM DISCHARGES IN THE TOKAMAK FUSION TEST REACTOR
}

S. J. Kilpatrick, C. S. Pitchera), M. G. Bell, D. M. Manes, D. K. Mansfield, I. Nyberg,

A. T. Ramsey, and B. C. Stratton

Princeton Plasma Physics Laboratory, Princeton, NJ 08543

\section{AESTRACT}

Langmuir probe measurements have been made across the plasma boundary in helium discharges of varying central electron densities ir the Tokamak Fusion Test Reactor (TFTR). Radial profiles of ion saturation current densily, $I_{S}$, electron density, $n_{\theta}$, and electron temperalure, $T_{\theta}$, were achieved by small displacements of the plasma relative to a slationary probe. Scrape-off lengths outside the plasma boundary decreased somewhat with increasing plasma density. Inside the plasma, $I_{S}, n_{\theta}$, and $T_{\theta}$ rose more steeply for the higher densities than the scrape-off lengths would predict. The floatirg potential, $V_{f}$, decreased sharply inside the boundary as expected, with some density depeildence. $I_{s}$ and $n_{\theta}$ increased with plasma density, while $T_{\theta}$ decreased a few eV. Measurements of $C$ II emission at the inner wall combined with probe data support a relatively constant edge $T_{\mathbf{\theta}}$ with increasing plasma density and a constant ratio between the fluxes of helium and carbon ions.

(Presented at the 36th American Vacuum Society National Symposium, Boston, 23 - 27 October 1989 , and accepted for publication in the Journal of Vacuum Science and Technology A.)

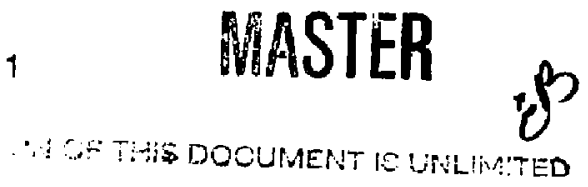




\section{INTRODUCTIOH}

Il is important to measure the edge plasma parameters at or inside the tokamak plasma boundary [last closed flux surface (LCFS)] in order to understand such issues as fuel recycling, graphite limiter conditioning, and impurity generation and transport, and to connect the edge measurements to thase of bulk plasma parameters. Several studies of this type have been made by edge probe diagnostics on large tokamaks [1-3].

In this paper we present the first Langmuir probe measurements inside the LCFS on the Tokamak Fusion Test Reactor (TFTR). A controlled sequence of Ohmic helium discharges forming a plasma density scan was investigated. A small vertical displacement of the plasma column during each discharge effectively scanned a stationary Langmuir probe across the LCFS. Radial profiles of ion saturation current densily, electron density, electron temperalure, and floating potential are reported. Their dependence on the plasma density is described. Simultaneous edge spectroscopy measurements are described and combined with the probe results.

\section{EXPERIMENTAL}

\section{A. Diagnostics}

The Langmuir probe measurements were made with the Combined Electrical and Sample Exposure Probe (CESEP) diagnostic, which has been described in detail elsewhere [4]. The probe enters the vacuum vessel from below the machine, at a major radius somewhat larger than the plasma center. The probe head was a $3.8 \mathrm{~cm}$ diameter cylinder of graphite with three $2.8 \mathrm{~mm}$ diameter graphite rods $6.4 \mathrm{~mm}$ in length protruding from the leading edge. Two were biased as a double probe with a sinusoidal voltage at $60 \mathrm{~Hz}$. The third measured the floating potential, $V_{f}$. Data were collected at $5 \mathrm{kHz}$ and analyzed [5] to yield electron densily, $n_{\theta}$, and electron temperature, $T_{\theta}$, for every cycle of the applied voltage. For the lowesl density plasmas, the gain circuit which was used unfortunately had high noise levels. Thus, only the ion saititation current density. $I_{5}$, could be extracted from these data during the plasma movement.

Measurements of C II emission were made with a multichannel visible spectrometer [6] mounted radially at the outer midplane, which has a poloidal array of views toward the axisymmetric graphite bumper limiter on the inner wall. The line-integral electron density was 
monitored and controlled by a ten-channel far-infrared laser interferometer [7] with signal feedback. The diagnostic path length for these plasmas was $160 \mathrm{~cm}$.

\section{B. Plasma conditions}

A series of eight consecutive helium discharges were made in this experiment, each with a constant line-integral electron density, incremented from $\mathrm{n}_{\theta} \mathrm{l}=1.4 \times 10^{15} \mathrm{~cm}^{-2}$ (pre-fill gas only for plasma formation) to $4.5 \times 10^{15} \mathrm{~cm}^{-2}$ over the course of the scan. The plasmas were run on the inner bumper limiter with major radius $\mathrm{R}=2.45 \mathrm{~m}$ and minor radius $\mathrm{a}=0.80 \mathrm{~m}$, with a toroidal field of $\mathrm{B}_{\mathrm{T}}=2.87 \mathrm{~T}$ and a plasma current $\mathrm{I}_{\mathrm{p}}=1.0 \mathrm{MA}$.

At $t=3.0$ sec after the slart of each discharge the plasina was slowly moved downward 5 $\mathrm{cm}(\Delta \mathrm{t}=0.4 \mathrm{sec})$ at constant major radius toward the probe 10 create radial profiles of electron density and temperature through the LCFS into the confined plasma. At $t=3.4 \mathrm{sec}$ the plasma was moved quickly ( $\Delta t=0.1 \mathrm{sec}$ ) back to its equilibrium position to avoid overheating the probe. The probe position was fixed for these plasmas and chosen to give as much plasma penetration as possible during the plasma movement phase while preserving the integrity of the Langmuir signals and minimizing the perturbation of the plasma.

The distance of the Langmuir elements from the LCFS throughout the plasma movement was calculated using a filament code and the plasma magnetics data. The probe position with respect to the vacuum vessel is known to within $\pm 3 \mathrm{~mm}$. According to the analysis, the LCFS at the bottom of the torus only moved $\sim 3.6 \mathrm{~cm}$, because the plasma was distorted somewhat as it was pressed down against the curved bumper limiter. The computed initial position of the centers of the Langmuir elements was $+0.9 \mathrm{~cm}$ (+ being defined as outside the plasma) with an absolute uncertainty of $+0.5 /-0.8 \mathrm{~cm}$ depending on which group of magnetic sensors were used. The relative movement of the plasma with respect to the probe is known to better than $-1 \mathrm{~mm}$. The probe floating potential measurements should also be an indicator of the LCFS. One would expect $V_{f}$ to change abruptly from values close to zero in the scrape-off layer to large negative values as the tip. penetrates the boundary and moves from a region where flux surfaces are shorted by the conducting limiter to a region which can support significant electric fields. Indeed, this was observed as shown below, and based on these measurements the initial probe position was +2.0 $\mathrm{cm} \pm 0.2 \mathrm{~cm}$. This value was used to set the distance scale in the following analyses. 


\section{RESULTS AND DISCUSSION}

\section{A. Langmulr probes}

Radial profiles of ion saturation current density (or equivalently ion flux density). Electron density, and electron temperature generated by the movement of the plasma toward the probe are shown in Fig. 1 for the range of plasma densities encountered. An ion mass $m_{j}=4$ and $T_{\theta}=T_{i}$ (where $T_{i}$ is the ion temperature) were assumed. All three parameters rose significantly in the $-3.6 \mathrm{~cm}$ which were explored. The noise level in the Langmuir probe current was significantly reduced at higher densities, as can be seen in Fig. 1 (a). A genile hump in $I_{s}$ and $n_{\theta}$ was observed at the approximate position of the plasma boundary for the higher plasma densities. Exponentials were fitted to the $I_{s}, n_{\theta}$, and $T_{\theta}$ curves for the data outside the LCFS $(d>0)$, and scrape-off lengths were calculated. These are plotted in Fig. 2 versus the line-integral censity. Note the gradual steepening of the scale lengths with plasma density. These Langmuir data at $n_{\theta} l=$ $1.4 \times 10^{15^{\prime}} \mathrm{cm}^{-2}$ are in general agreement with earlier probe profiles [3] up to the LCFS at the outer midplane in 1.0 MA deuterium prefill gas-only plasmas $\left.\left(n_{\theta}\right)=1.3 \times 10^{15} \mathrm{~cm}^{-2}\right)$.

Inside the plasma, $I_{s}, n_{e}$, and $T_{\theta}$ rose more steeply for the higher densities than the scrape-off lengths would predict, according to Fig.1. The edge density rise has been compared to Abel-inverted [8] density profiles near the plasma periphery from the multichannel interterometer. There is good agreement between the two measurements, aithough at the LCFS the errors in the interferometer density values are approximately $+1.10 \%$ with a spatial resolution of $\sim 3-5 \mathrm{~cm}$.

Figure 3 shows the radial dependence of the floating potential (measured with respect to the vacuum vessel) tor these plasmas. The sharp decrease in these curves always occurred at the same position and has been used to determine the location of the LCFS $(d=0)$. Some variation of the profiles with plasma density was observed. Outside the plasma boundary, $V_{\text {, was greater than }}$

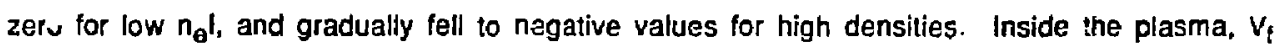
decreased more sharply with decreasing plasma density.

The plasma density dependence of the Langmuir data is highlighted in Fig. 4. $I_{s}, n_{\theta}$, and $T_{\theta}$ are plotted against $n_{\theta} l$ for three radial positicns of interest: before the plasma movement at $d=$ 
$+2.0 \mathrm{~cm}$ (with data averaged over several cycles of the applied voltage), at the LCFS (d =0), and approximately at the furthest penetration into the plasma, $\mathrm{d}=-1.5 \mathrm{~cm}$. There is a density dependence which changes somewhat with radial position. The edge density dala in Fig. $4(\mathrm{~b})$ for $d$ $=+2.0 \mathrm{~cm}$ was fitted to a power law dependence, $n_{\theta}{ }^{\text {edge }} \alpha\left(n_{\theta} \mid\right)^{0.7}$. The data can be fitted equally well to an offset linear dependence. Previous measurementis on TFTR in deuterium plasmas showed an exponential dependence [9] for Ohmic discharges on a single oulboard limiter and a linear dependence [10] during neutral-beam-heated discharges on the bumper limiter, while Onmic deuterium data [1] from the Joint European Torus (JET) suggest $n_{\theta}(a) \alpha n_{\theta}{ }^{k}$, where $k$ is usually 1 to 2 .

\section{B. Spectroscopy}

Measuremenis of the C II intensity from the visible spectrometer as a function of poloidal angle from the plasina center are displayed in Fig. 5(a), with the data at $0^{\circ}$ coming lrom the plasma contact point at the center of the bumper limiter and at $75^{\circ}$ coming from a region above the top edge of the limiter. The C II radiation originates primarily from the boundary plasma in front of the limiter, where electron temperatures are only a few tens of eV. As one would expect, the peaks in these curves occur at the poloidal region on the limiler for which the incident particle flux and power flux are normally the highest [11], several degrees above the midplane. Note that the shape narrows somewhat at high plasma density. This is in qualitative agreement with the lower particle flux scrape-off lengths observed at higher densities.

The spatially integrated $\sigma$ II intensities are plotted against the spatially integrated ion saturation current densities for the density scan in Fig. 5(b). The C II integral was formed by summing the intensities from the five channels, while the $I_{s}$ integral simply sums the measured $I_{s}$ values for $d>0$. A linear dependence is observed. Such behavior would be expected if the following remained constant during the density scan: (1) the physical sputtering yields for helium and carbon ions (the oxygen content is negligible in these discharges), (2) the photon efficiency for the radiating $C$ II ions, and (3) the distribution of charge states in the boundary plasma. Both the sputtering yields [12] and the photon efficiency [13] depend snly on the electron temperature in the boundary plasma, which from the probe measurements varies only modestly during the density scan, Fig. 4 (c). As for the charge state distribution, both experiment and theory [14] predict a charge state distribution which is relatively insensitive to boundary plasma conditions. Thus, the linear relationship shown in Fig. $\mathbf{5 ( b )}$ is consistent with a constant 
sputtering yield for ions incident on the graphite bumper limiter and a constant ratio between the fluxes of helium and carbon ions.

\section{CONCLUSIONS}

Langmuir probe measurements have been made across the LCFS in helium discharges in TFTR. Scrape-off lengths outside the plasma boundary decreased somewhat with increasing plasma density to $\lambda_{n} \approx \lambda_{T}=5 \mathrm{~cm}$ and $\lambda_{p}=3.2 \mathrm{~cm}$ at $n_{\theta} l=4.5 \times 10^{15} \mathrm{~cm}^{-2}$. Inside the plasma, $I_{s}, n_{\theta}$, and $T_{\theta}$ rose more sleeply for the higher densities than the scrape-off lengths would predict. The floating potential $V_{f}$ decreased sharply inside the boundary as expected, with some derisity dependence. Outside the boundary, $n_{\theta}$ increased as $\left(n_{\theta} \mid\right)^{0.7}$. Meanwhile, $T_{\theta}$ dropped a few $\mathrm{eV}$ and $\mathrm{I}_{\mathrm{s}}$ steadily increased. Spatially integrated $\mathrm{C}$ II intensities were found to be proportional to the spatially integrated $I_{s}$ data through the density scan, consistent with a relatively constant edge $T_{\theta}$ and a constant ratio between the fluxes of helium and carbon ions.

\section{ACKNOWLEDGMENTS}

This work was supported by the U. S. Department of Energy Contract No. DE-AG02-76-CHO-3073. 


\section{BEEERENCES}

a)Permanent address: Canadian Fusion Fuels Technology Project, Toronto, Canada

1. S. K. Erents, J. A. Tagle, G. M. McCracken, P. C. Stangeby, and L. de Kock, Nucl. Fusion 28, 1209 (1988).

2. N. L. Vasin, V. A. Vershkov, S. A. Grashin, A. A. Medvedev, A. Yu. Pigarov etal. J. Nucl. Mater. 162-164, 300 (1989).

3. S. J. Kilpatrick, D. M. Manos, M. Diesso, and K. Roayaie, Bull. Am. Phys. Soc. 32, 1806 (1987).

4. R. Mastronardi, R. Cabral, and D. Manos, in Proc. 10th_Symp. on Fusion Engineering, Philadelphia, 1983 (IEEE, New York, 1983), p. 1507.

5. D. M. Manos and G. M. McCracken, in Rlasma Wall_nteractions in Controlled_Fusion Devices, edited by R. Behrisch and D. Post (Plenum, New York, 1986).

6. A. T. Ramsey and S. L. Turner, Rev. Sci. Instrum. 58, 1211 (1987).

7. D. K. Mansfield, H. K. Park, L. C. Johnson, H. M. Anderson, R. Chouinard et al., Appl. Oplics 26. 4469 (19B7).

8. H. K. Park, Plasma Phys. Controlled Fusion 31, 2035 (1989).

9. S. J. Kilpatrick, D. M. Manos, R. V. Budny, P. C. Stangeby, R. S. Ritterel_al, J. Vac. Sci. Technol. A4, 1817 (1986).

10. D. M. Manos, S. J. Kilpatrick, M. G. Bell, R. V. Budny, and M. Ulrickson, J. Nucl. Mater. 162-164, 251 (1989).

11. R. T. McGrath and J. N. Brooks, J. Nucl. Mater. 162-164, 350 (1989).

12. G. M. McCracken and P. E. Stott, Nucl. Fusion 19, 889 (1979).

13. K. H. Behringer, J. Nucl. Mater. 145-147, 145 (1987).

14. G. F. Matthews, J. Nucl. Mater. 162-164, 38 (1989). 


\section{EIGURE CAPTIONS}

Fig. 1 Radial profiles of (a) ion saturation current density, (b) electron density, and (c) electron temperature for varying line-integral electron densities, $n_{\boldsymbol{e}} \mathbf{l}$. Negative values of $d$, the distance from the LCFS, are defined as inside the plasma. The error bars for $n_{\theta}$ and $T_{\theta}$ represent the average $95 \%$ confidence intervals for the fitted data.

Fig. 2 Plasma density variation of the scrape-olf lengths $\lambda_{p}, \lambda_{n}$, and $\lambda_{T}$, which were calculaied from the data outside the LCFS in Fig. 1.

Fig. 3 The radial dependence of the floating potential imeasured with resfect to the vacuum vessel) for varying plasma densities, $n_{\theta}$.

Fig. 4 Plasma density variation of (a) the ion saturation current density, (b) the electron densily, and (c) the electron temperature tor three radial positions of interest. The error bars for $n_{0}$ and $\tau_{e}$ represent the $95 \%$ confidence intervals for each fit.

Fig. 5 (a)Measurements of the C I' intensity near the inner wall along five poloidally spaced sightlines of a visible spectrcmeter array. The poloidal angle is measured with respect to the plasma center, with $0^{0}$ being the bumper limiter midplane. (b)The spatially integrated C II intensity $\%$ sus the spatially integrated ion saturation current density for the plasma density scan. 

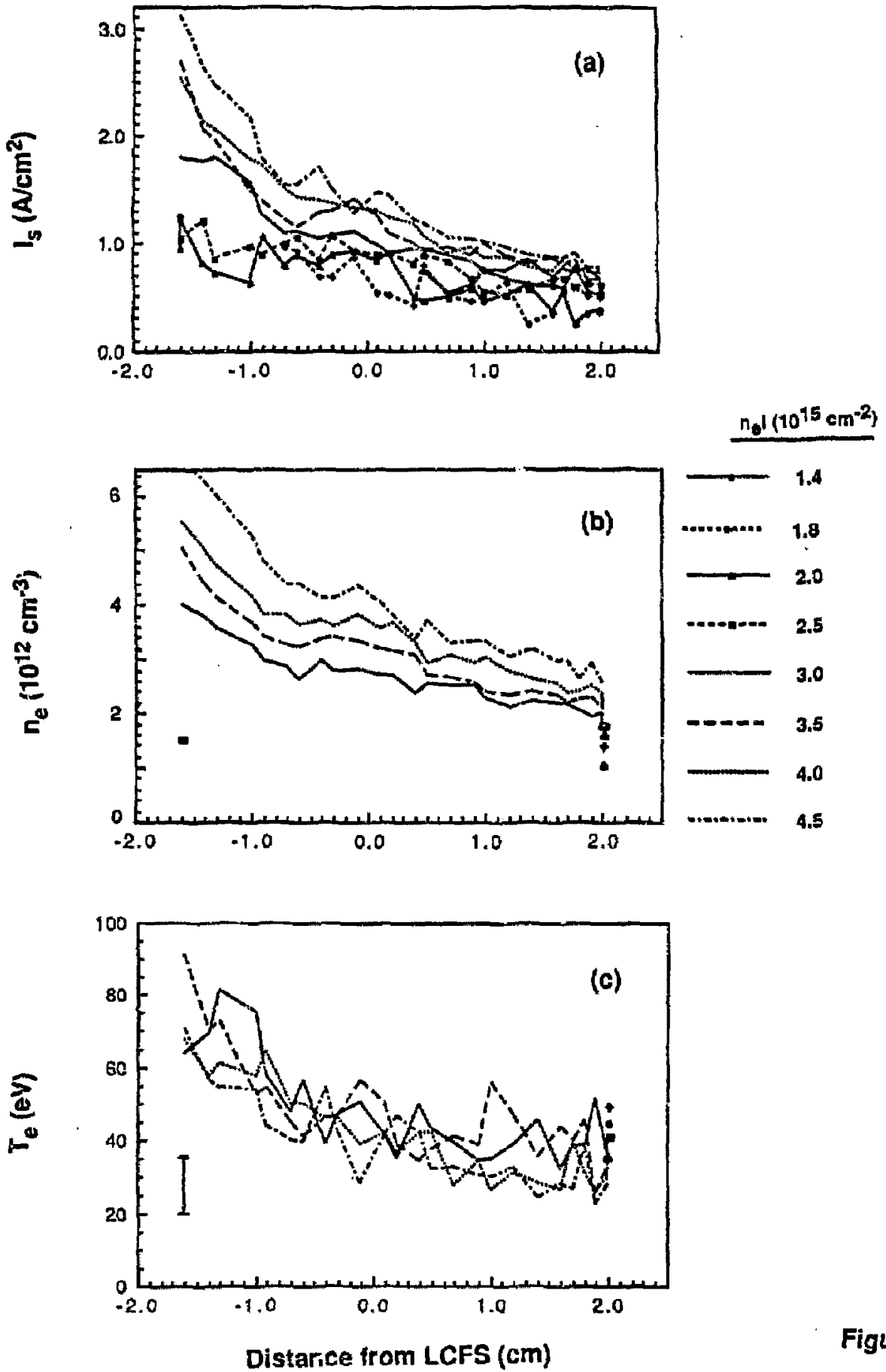

Figure 1 


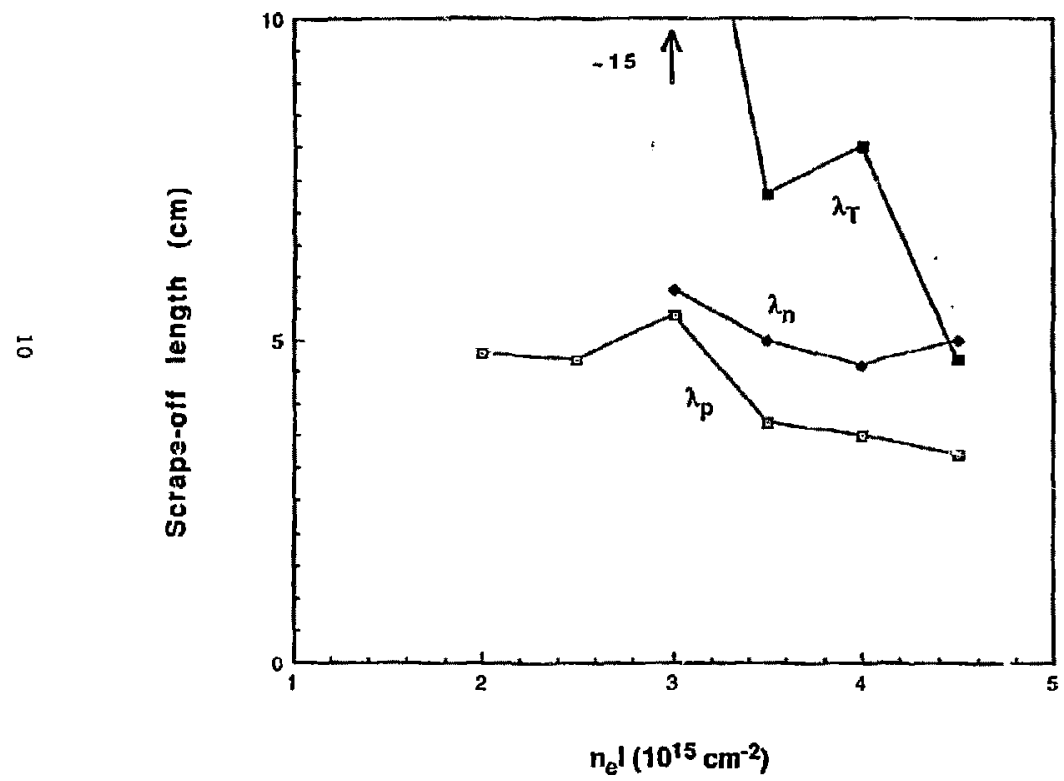

Figure 2

$=\quad$. 
Floating potential, $V_{f}$ (V.)

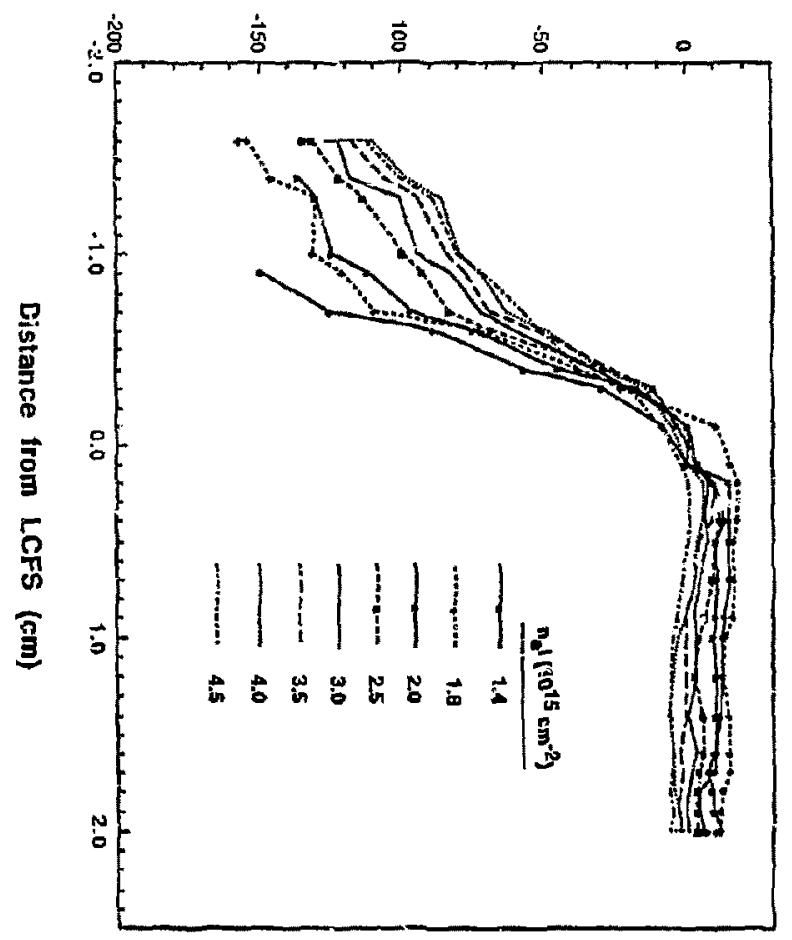

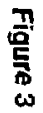



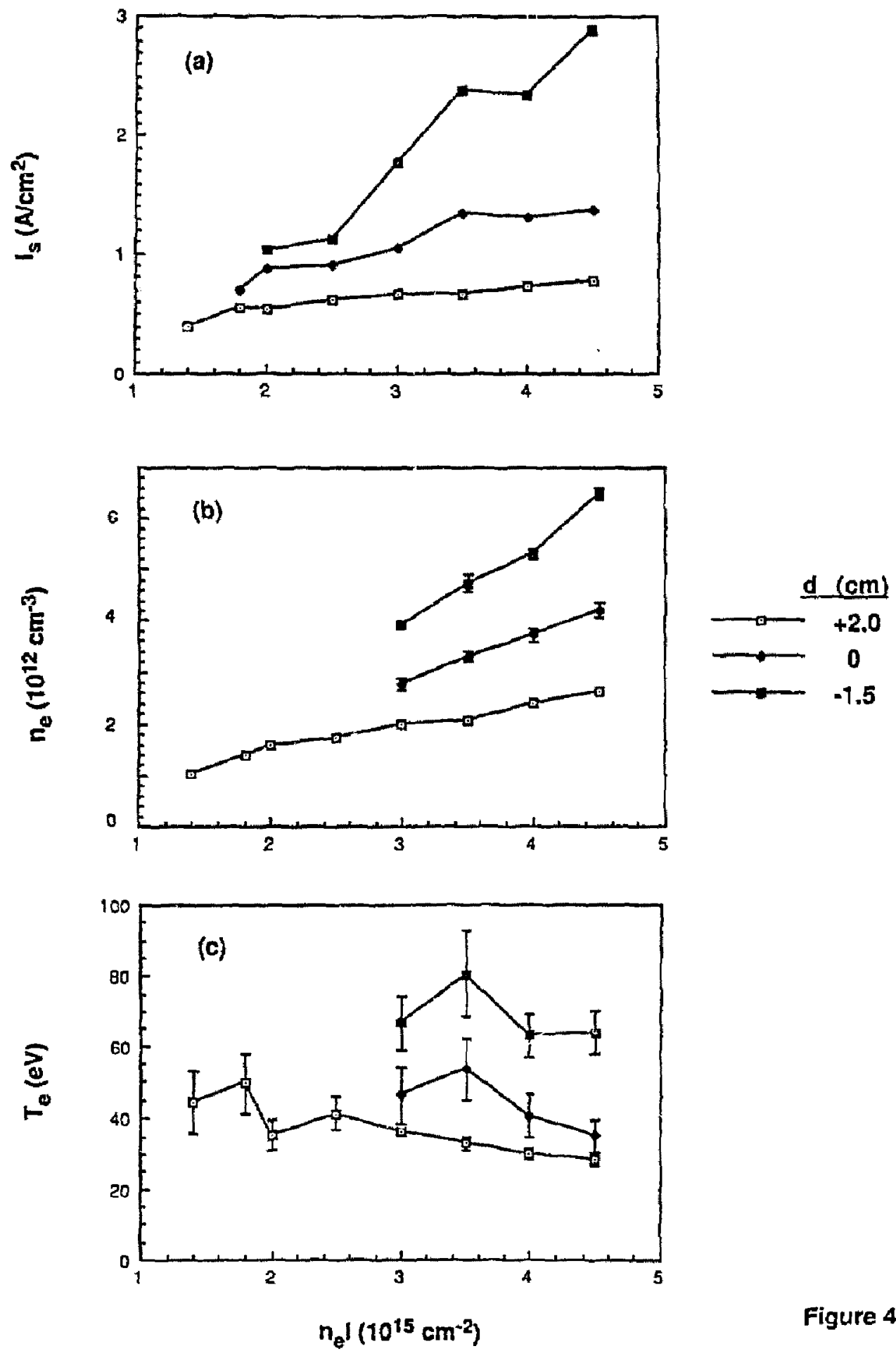

Figure 4 
Cll Intensity (arb. units)

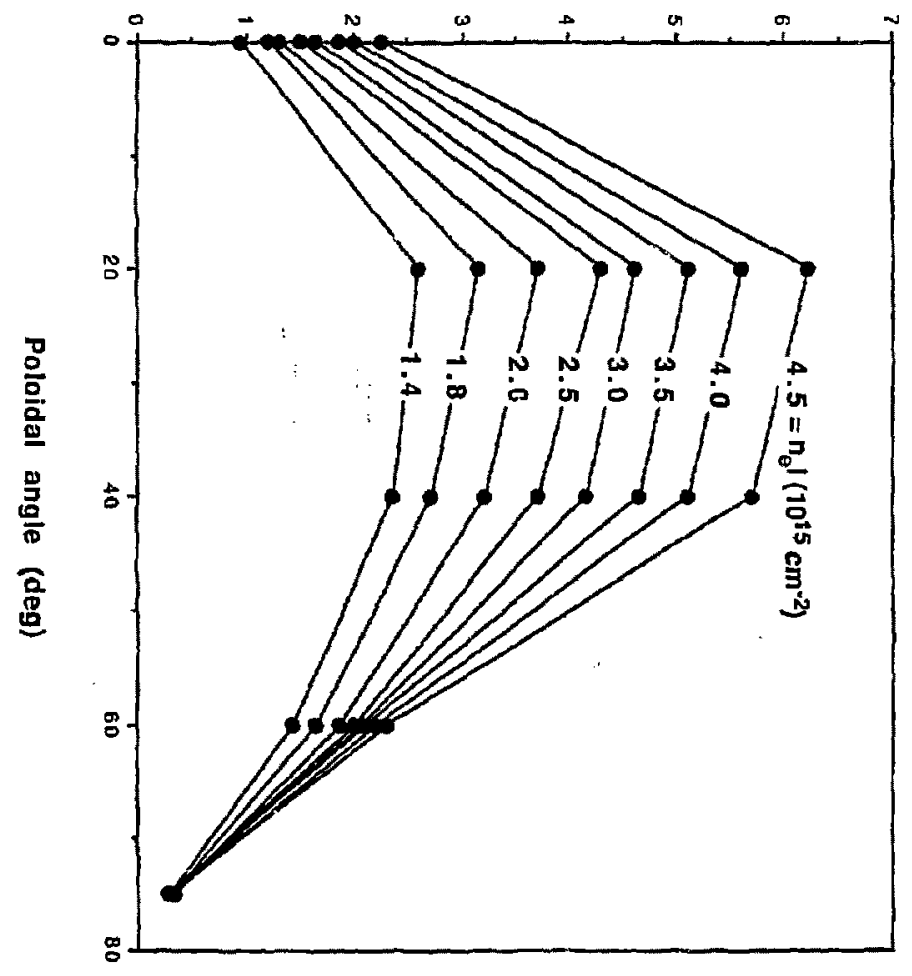

$\frac{\pi}{6}$
을
늘 
Integrated cll intensity (a.u.)

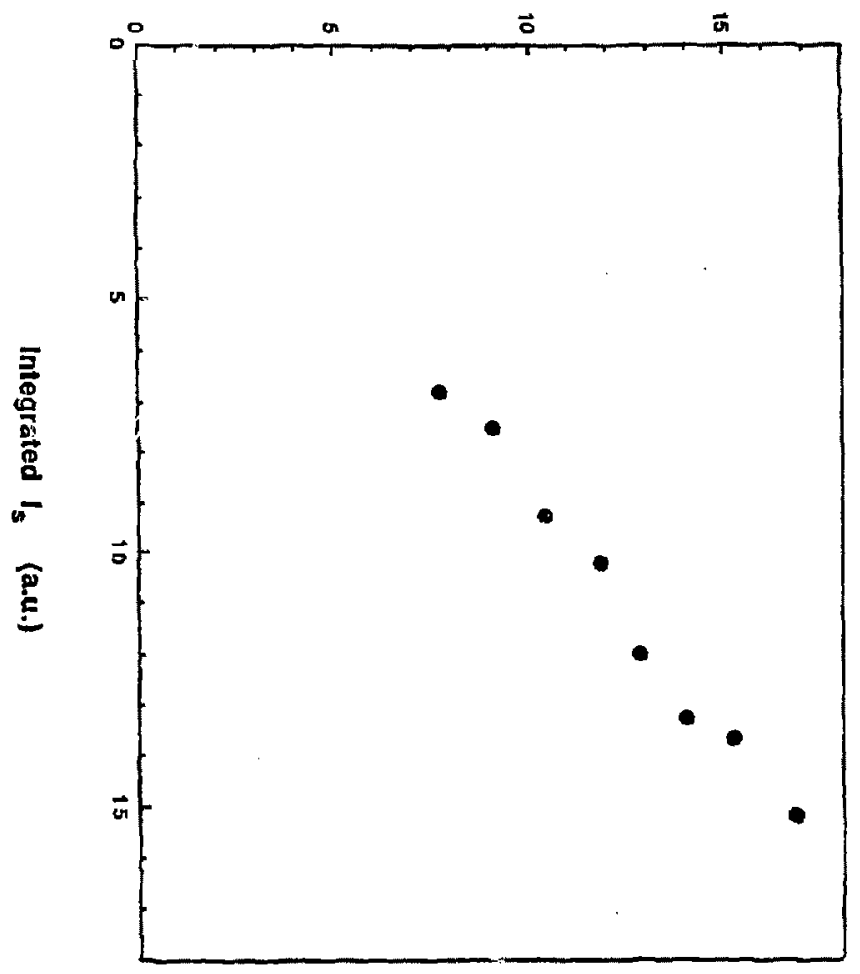

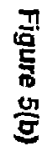

